Vietnam Journal of Mechanics, VAST, Vol.43, No. 1 (2021), pp. 55-77

DOI: https://doi.org/10.15625/0866-7136/15467

\title{
NONLINEAR VIBRATION OF NONLOCAL STRAIN GRADIENT NANOTUBES UNDER LONGITUDINAL MAGNETIC FIELD
}

\author{
N. D. Anh ${ }^{1,2}$, D. V. Hieu ${ }^{3, *}$ \\ ${ }^{1}$ Institute of Mechanics, VAST, Hanoi, Vietnam \\ ${ }^{2}$ VNU University of Engineering and Technology, Hanoi, Vietnam \\ ${ }^{3}$ Thai Nguyen University of Technology, Vietnam \\ *E-mail: hieudv@tnut.edu.vn
}

Received: 20 September 2020 / Published online: 10 January 2021

\begin{abstract}
The nonlinear free vibration of embedded nanotubes under longitudinal magnetic field is studied in this paper. The governing equation for the nanotube is formulated by employing Euler-Bernoulli beam model and the nonlocal strain gradient theory. The analytical expression of the nonlinear frequency of the nanotube is obtained by using Galerkin method and the equivalent linearization method with the weighted averaging value. The accuracy of the obtained solution has been verified by comparison with the published solutions and the exact solution. The influences of the nonlocal parameter, material length scale parameter, aspect ratio, diameter ratio, Winkler parameter and longitudinal magnetic field on the nonlinear vibration responses of the nanotubes with pinned-pinned and clamped-clamped boundary conditions are investigated and and discussed.
\end{abstract}

Keywords: nonlinear vibration, carbon nanotube, nonlocal strain gradient, magnetic field, Galerkin method, equivalent linearization, weighted averaging.

\section{INTRODUCTION}

First discovered in 1991 by Iijima [1], carbon nanotubes (CNTs) show many advantages compared to conventional steel tubes. With theirs advantages and small sizes, CNTs have many applications such as nanoactuator [2], nano-electromechanical systems (NEMS) [3, 4], nano-devices for electronics [5,6], nano-medicine [7], or nano-devices for conveying fluid and gas storage $[8,9]$.

Researching the mechanical behavior of CNTs is an extremely important problem and attracts many interested scientists. Unlike macro-sized tubes, the size-dependent effect plays an important role in the response of CNTs. Some elasticity theories have been proposed to study the size-dependent effect on the mechanical response of micro-/nanostructures such as Eringen's nonlocal elasticity theory $[10,11]$, the strain gradient theory

(C) 2021 Vietnam Academy of Science and Technology 
(SGT) $[12,13]$, and the modified couple stress theory (MCST) [14]. To date, many works related to the static and dynamic responses of CNTs have been published using these higher-order elasticity theories. Nonlinear free vibration responses of the single-walled carbon nanotubes (SWCNTs) were reported by Yang et al. [15] using the Timoshenko beam theory and Eringen's nonlocal elasticity theory. The effects of nonlocal parameter, length and radius of the SWCNTs with different boundary conditions on the nonlinear free vibration behaviors of SWCNTs were examined in this work. Narendar et al. [16] studied the wave propagation problem in the SWCNTs under longitudinal magnetic field based on Eringen's nonlocal elasticity theory and the Euler-Bernoulli beam theory. The wave method was employed by Zhang et al. [17] to analyze the nonlinear free vibration of the fluid-conveying SWCNTs based on Eringen's nonlocal elasticity theory. Based on the Euler-Bernoulli beam theory and Eringen's nonlocal elasticity theory, Wang and Li [18] investigated the nonlinear free vibration of the SWCNTs with the viscous damping effect. Zhen and Fang [19] used the Lindstedt-Poincaré method to investigate the nonlinear vibration of the fluid-conveying SWCTNs under harmonic excitation. Nonlinear vibration of the embedded SWCNTs was reported by Valipour et al. [20] using Eringen's nonlocal elasticity theory and the parameterized perturbation method. Goughari et al. [21] studied effects of magnetic-fluid flow on the instability of the fluid-conveying SWCNTs under the magnetic field. Free vibration of the SWCNTs resting on the exponentially varying elastic foundation was examined by Chakraverty and Jena using Eringen's nonlocal elasticity theory [2]. Based on the MCST and the Euler-Bernoulli beam theory, Wang [22] investigated the size-dependent vibration responses of the fluid-conveying microtubes. Flexural size-dependent vibrations of the microtubes conveying fluid was carried out by Wang et al. [23] utilizing the MCST. Based on the MCST, Tang et al. [24] developed a nonlinear model to study the size-dependent vibration of the curved microtubes conveying fluid. Xia and Wang [25] studied vibration and stability behaviors of the microscale pipes conveying fluid based on Timoshenko beam theory and the MCST. Based on the second SGT, vibration and stability behaviors of the SWCNTs conveying fluid were presented by Ghazavi et al. [26].

In 2015, the nonlocal parameter and the material length scale parameter were incorporated into a generalized elasticity theory. Lim et al. [27] introduced the nonlocal strain gradient theory (NSGT) describing two entirely different physical characteristics of materials and structures at nanoscale. Many works related to behaviors of micro-/nanobeams and micro-/nano-tubes have been published by using the NSGT. Using the NSGT and the Euler-Bernoulli beam model, Simsek investigated nonlinear free vibration response of a functionally graded (FG) nanobeam [28]. Bending, buckling, and vibration responses of viscoelastic FG curved nanobeam resting on an elastic foundation were studied by Allam and Radwan using the NSGT [29]. Nonlinear vibration of the electrostatic FG nano-resonator considering the surface effects was investigated by Esfahani et al. [30] employing the Euler-Bernoulli beam theory and the NSGT. Based on the NSGT, Dang et al. [31] analyzed nonlinear vibration behavior of nanobeam under electrostatic force. A nonlocal strain gradient Timoshenko beam model was developed by Bahaadini et al. [32] to analyze the vibration and instability responses of the SWCNTs conveying nanoflow. Using the NSGT and the shear deformation beam model, Malikan et al. [33] studied the 
damped forced vibration of the SWCNTs resting on viscoelastic foundation in thermal environment. Vibration behaviors of porous nanotubes were investigated by She et al. [34] utilizing the NSGT and the refined beam model. Atashafrooz et al. [35] analyzed effects of nonlocal, strain gradient and surface stresses on vibration and instability behaviors of the SWCNTs conveying nanoflow by using the NSGT and the Euler-Bernoulli beam theory. Based on the NSGT, Li et al. [36] studied size-dependent effects on critical flow velocity of microtubes conveying fluid; both Timoshenko and Euler-Bernoulli beam models were considered in this work. Nonlinear forced vibration of the SWCNTs was examined by Ghayesh and Farajpour using the NSGT and the Euler-Bernoulli beam model [37]. The coupled nonlinear mechanical behavior of nonlocal strain gradient SWCNTs subjected to distributed load was investigated by Ghayesh and Farajpour [38]. Dynamic response of the viscoelastic SWCNTs conveying fluid with uncertain parameters and under random excitation was analyzed by Azrar et al. [39] by combining the NSGT and the EulerBernoulli beam theory. The problem of wave propagation in the SWCNTs also attracted many authors [40-42].

According to authors' knowledge, until now, nonlinear vibration response of the nonlocal strain gradient SWCNTs under magnetic field has not yet announced. Thus, in this paper, authors focus on studying the effect of magnetic field on the nonlinear vibration response of the SWCNTs based on the NSGT. Using the equivalent linearization method with the weighted averaging value [43-49], expressions of the nonlinear frequencies of the SWCNTs resting on the elastic foundation and under the longitudinal magnetic field are given in the analytical forms. Effects of the nonlocal parameter, material length scale parameter, aspect ratio, diameter ratio, magnetic field and elastic foundation on the nonlinear vibration behaviors of the SWCNTs with different boundary conditions are investigated and discussed in this work.

\section{MODEL AND FORMULATIONS}

Considering a SWCNT made of the homogeneous material as shown in Fig. 1. The nanotube has the length $L$, the inner diameter $d$, the outer diameter $D$, the mass density $\rho$ and the Young's modulus $E$. The nanotube rests on a linear elastic foundation with a coefficient of $k_{w}$ (the Winkler layer) and is subjected to a longitudinal magnetic field.

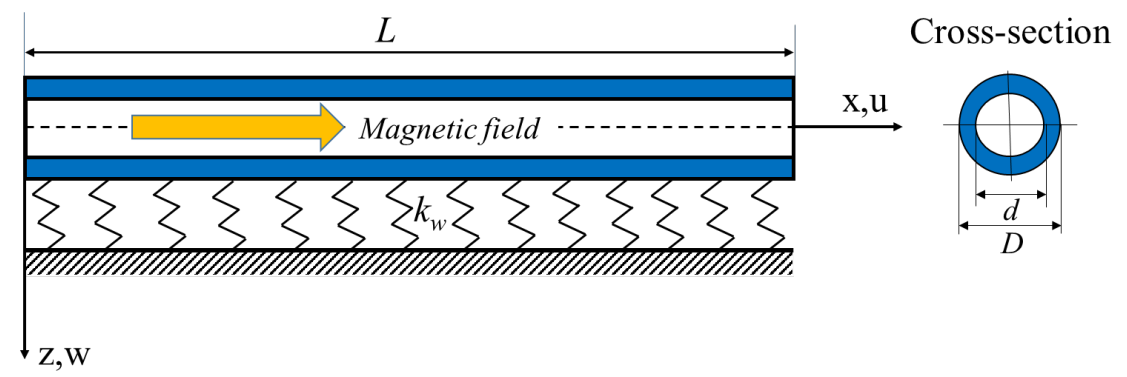

Fig. 1. Model of the SWCNT resting on an elastic foundation and under longitudinal magnetic field 
Based on the Euler-Bernoulli beam theory and the von-Karmán's nonlinear straindisplacement relationship, the strain-displacement relationship for the nanotube takes a form

$$
\varepsilon_{x x}=\frac{\partial u(x, t)}{\partial x}+\frac{1}{2}\left(\frac{\partial w(x, t)}{\partial x}\right)^{2}-z \frac{\partial^{2} w(x, t)}{\partial x^{2}}
$$

where $u(x, t)$ and $w(x, t)$ are the axial and transverse displacements, respectively; and $t$ is time.

According to the NSGT proposed by Lim et al. [27], the strain energy can be expressed as

$$
U=\frac{1}{2} \int_{V}\left(\sigma_{x x} \varepsilon_{x x}+\sigma_{x x}^{(1)} \nabla \varepsilon_{x x}\right) d V,
$$

where $\sigma_{x x}$ and $\sigma_{x x}^{(1)}$ are the classical and higher-order stresses, respectively, which are defined as

$$
\begin{gathered}
\sigma_{x x}=\int_{0}^{L} E \alpha_{0}\left(x, x^{\prime}, e_{0} a\right) \varepsilon_{x x}^{\prime}\left(x^{\prime}\right) d x^{\prime}, \\
\sigma_{x x}^{(1)}=l^{2} \int_{0}^{L} E \alpha_{1}\left(x, x^{\prime}, e_{1} a\right) \varepsilon_{x x}^{\prime}\left(x^{\prime}\right) d x^{\prime}, \\
t_{x x}=\sigma_{x x}-\nabla \sigma_{x x}^{(1)},
\end{gathered}
$$

herein, $\nabla=\partial / \partial x$ represents the differential operator; $t_{x x}$ is the total stress; $\alpha_{0}$ and $\alpha_{1}$ are two nonlocal kernel functions; $e_{0} a$ and $e_{1} a$ denote the nonlocal parameters; $l$ is the material length scale parameter. With assuming that $e_{0} a=e_{1} a=e a$, the general nonlocal strain gradient constitutive equation for the nanotube can be presented as [27]

$$
\left[1-(e a)^{2} \nabla^{2}\right] t_{x x}=E\left(1-l^{2} \nabla^{2}\right) \varepsilon_{x x},
$$

where $\nabla^{2}=\partial^{2} / \partial x^{2}$ is the Laplacian operator. The Hamilton's principle is used to derive the equation of motion for the nanotube. From Eqs. (1) and (2), the virtual strain energy of the nanotube can be expressed as

$$
\begin{aligned}
\delta U= & \int_{0}^{L}\left\{N_{x x} \delta\left[\frac{\partial u}{\partial x}+\frac{1}{2}\left(\frac{\partial w}{\partial x}\right)^{2}\right]-M_{x x} \delta\left(\frac{\partial^{2} w}{\partial x^{2}}\right)\right\} d x+\left.\left\{N_{x x}^{(1)} \delta\left[\frac{\partial u}{\partial x}+\frac{1}{2}\left(\frac{\partial w}{\partial x}\right)^{2}\right]-M_{x x}^{(1)} \delta\left(\frac{\partial^{2} w}{\partial x^{2}}\right)\right\}\right|_{0} ^{L} \\
= & {\left.\left[N_{x x} \delta u\right]\right|_{0} ^{L}-\int_{0}^{L} \frac{\partial N_{x x}}{\partial x} \delta u d x+\left.\left[N_{x x} \frac{\partial w}{\partial x} \delta w\right]\right|_{0} ^{L}-\int_{0}^{L} \frac{\partial}{\partial x}\left[N_{x x} \frac{\partial w}{\partial x}\right] \delta w d x-\left.\left[M_{x x} \delta\left(\frac{\partial w}{\partial x}\right)\right]\right|_{0} ^{L}+\left.\left[\frac{\partial M_{x x}}{\partial x} \delta w\right]\right|_{0} ^{L} } \\
& -\int_{0}^{L} \frac{\partial^{2} M_{x x}}{\partial x^{2}} \delta w d x+\left.\left\{N_{x x}^{(1)}\left[\delta\left(\frac{\partial u}{\partial x}\right)+\frac{\partial w}{\partial x} \delta\left(\frac{\partial w}{\partial x}\right)\right]-M_{x x}^{(1)} \delta\left(\frac{\partial^{2} w}{\partial x^{2}}\right)\right\}\right|_{0} ^{L}
\end{aligned}
$$


where $N_{x x}$ is the axial force resultant, $N_{x x}^{(1)}$ is nonclassical axial force resultant, $M_{x x}$ is the bending moment resultant, and $M_{x x}^{(1)}$ is the nonclassical moment resultant, these resultants are defined by

$$
N_{x x}=\int_{A} t_{x x} d A, N_{x x}^{(1)}=\int_{A} \sigma_{x x}^{(1)} d A, M_{x x}=\int_{A} z t_{x x} d A, M_{x x}^{(1)}=\int_{A} z \sigma_{x x}^{(1)} d A .
$$

The virtual kinetic energy of the nanotube is given by

$$
\delta K_{e}=\rho A \int_{0}^{L}\left[\frac{\partial u}{\partial t} \delta\left(\frac{\partial u}{\partial t}\right)+\frac{\partial w}{\partial t} \delta\left(\frac{\partial w}{\partial t}\right)\right] d x,
$$

where $A$ is the cross-section area of the nanotube. The virtual external work is

$$
\delta W_{e}=\int_{0}^{L}(q \delta w) d x
$$

In this work, the external forces are caused by the linear elastic foundation and the longitudinal magnetic field

$$
q=q_{e}+q_{m}
$$

where the external force caused by the linear elastic layer can be expressed as

$$
q_{e}=-k_{w} w
$$

and the external force due to the longitudinal magnetic field is $[41,42]$

$$
q_{m}=\eta A H_{x}^{2} \frac{\partial^{2} w}{\partial x^{2}}
$$

in which, $\eta$ is the magnetic permeability and $H_{x}$ is the component of the longitudinal magnetic field vector exerted on the nanotube in the $x$-direction.

Substituting Eqs. (12) and (13) into Eq. (10), one gets

$$
\delta W_{e}=\int_{0}^{L}\left[\left(-k_{w} w+\eta A H_{x}^{2} \frac{\partial^{2} w}{\partial x^{2}}\right) \delta w\right] d x .
$$

The Hamilton's principle is employed to derive the equation of motion for the nanotube, this principle states that

$$
\int_{0}^{t}\left(\delta K_{e}+\delta W_{e}-\delta U\right) d t=0
$$

Substituting Eqs. (7), (9) and (14) into Eq. (15); then using integration by parts, and collecting the coefficients of $\delta u$ and $\delta w$, one gets the equations of motion

$$
\frac{\partial N_{x x}}{\partial x}=\rho A \frac{\partial^{2} u}{\partial t^{2}}
$$




$$
\frac{\partial^{2} M_{x x}}{\partial x^{2}}+\frac{\partial}{\partial x}\left(N_{x x} \frac{\partial w}{\partial x}\right)-k_{w} w+\eta A H_{x}^{2} \frac{\partial^{2} w}{\partial x^{2}}=\rho A \frac{\partial^{2} w}{\partial t^{2}} .
$$

and the boundary conditions at $x=0$ and $x=L$ as

$$
\begin{aligned}
\delta u: & N_{x x}=0 \text { or } u=0 \\
\delta\left(\frac{\partial u}{\partial x}\right): & N_{x x}^{(1)}=0 \text { or } \frac{\partial u}{\partial x}=0, \\
\delta w: & \frac{\partial M_{x x}}{\partial x}+N_{x x} \frac{\partial w}{\partial x}=0 \text { or } w=0, \\
\delta\left(\frac{\partial w}{\partial x}\right): & M_{x x}-N_{x x}^{(1)} \frac{\partial w}{\partial x}=0 \text { or } \frac{\partial w}{\partial x}=0, \\
\delta\left(\frac{\partial^{2} w}{\partial x^{2}}\right): & M_{x x}^{(1)}=0 \text { or } \frac{\partial^{2} w}{\partial x^{2}}=0 .
\end{aligned}
$$

Note that the boundary conditions (18)-(22) will be satisfied in only one way for any specific support conditions of the nanotube $[36,50]$.

From Eqs. (1) and (6), one obtains

$$
\left[1-(e a)^{2} \nabla^{2}\right] t_{x x}=E\left(1-l^{2} \nabla^{2}\right)\left[\frac{\partial u}{\partial x}+\frac{1}{2}\left(\frac{\partial w}{\partial x}\right)^{2}-z \frac{\partial^{2} w}{\partial x^{2}}\right] .
$$

Considering Eq. (8), Eq. (23) leads to

$$
\begin{aligned}
& {\left[1-(e a)^{2} \frac{\partial^{2}}{\partial x^{2}}\right] N_{x x}=E A\left(1-l^{2} \frac{\partial^{2}}{\partial x^{2}}\right)\left[\frac{\partial u}{\partial x}+\frac{1}{2}\left(\frac{\partial w}{\partial x}\right)^{2}\right],} \\
& {\left[1-(e a)^{2} \frac{\partial^{2}}{\partial x^{2}}\right] M_{x x}=-E I\left(1-l^{2} \frac{\partial^{2}}{\partial x^{2}}\right) \frac{\partial^{2} w}{\partial x^{2}} .}
\end{aligned}
$$

where $I=\int_{A} z^{2} d A$ is the moment of inertia of the nanotube. Using Eqs. (16), (17), (24) and (25), the expressions of the axial force and bending moment resultants can be get as

$$
\begin{aligned}
& N_{x x}=E A\left(1-l^{2} \frac{\partial^{2}}{\partial x^{2}}\right)\left[\frac{\partial u}{\partial x}+\frac{1}{2}\left(\frac{\partial w}{\partial x}\right)^{2}\right]+(e a)^{2}\left(\rho A \frac{\partial^{3} u}{\partial x \partial t^{2}}\right) \\
& M_{x x}=-E I\left(1-l^{2} \frac{\partial^{2}}{\partial x^{2}}\right) \frac{\partial^{2} w}{\partial x^{2}}+(e a)^{2}\left[\rho A \frac{\partial^{2} w}{\partial t^{2}}-\frac{\partial}{\partial x}\left(N_{x x} \frac{\partial w}{\partial x}\right)+k_{w} w-\eta A H_{x}^{2} \frac{\partial^{2} w}{\partial x^{2}}\right] .
\end{aligned}
$$

Substituting Eqs. (26) and (27) into Eqs. (16) and (17), one gets the differential equations of motion for the nanotube as

$$
E A \frac{\partial}{\partial x}\left\{\left(1-l^{2} \frac{\partial^{2}}{\partial x^{2}}\right)\left[\frac{\partial u}{\partial x}+\frac{1}{2}\left(\frac{\partial w}{\partial x}\right)^{2}\right]\right\}-\rho A \frac{\partial^{2}}{\partial t^{2}}\left[1-(e a)^{2} \frac{\partial^{2} w}{\partial x^{2}}\right]=0
$$




$$
\begin{aligned}
& -E I\left(1-l^{2} \frac{\partial^{2}}{\partial x^{2}}\right) \frac{\partial^{4} w}{\partial x^{4}}+\frac{\partial}{\partial x}\left(N_{x x} \frac{\partial w}{\partial x}\right)-(e a)^{2} \frac{\partial^{3}}{\partial x^{3}}\left(N_{x x} \frac{\partial w}{\partial x}\right)-\rho A \frac{\partial^{2}}{\partial t^{2}}\left[w-(e a)^{2} \frac{\partial^{2} w}{\partial x^{2}}\right] \\
& -k_{w}\left[w-(e a)^{2} \frac{\partial^{2} w}{\partial x^{2}}\right]+\eta A H_{x}^{2} \frac{\partial^{2}}{\partial x^{2}}\left[w-(e a)^{2} \frac{\partial^{2} w}{\partial x^{2}}\right]=0 .
\end{aligned}
$$

With assuming that the axial inertia term in Eq. (28) is neglected, the axial force resultant can be achieved as $[28,37]$

$$
N_{x x}=\frac{E A}{2 L} \int_{0}^{L}\left(\frac{\partial w}{\partial x}\right)^{2} d x-\frac{E A}{L} l^{2} \int_{0}^{L}\left[\frac{\partial w}{\partial x} \frac{\partial^{3} w}{\partial x^{3}}+\left(\frac{\partial^{2} w}{\partial x^{2}}\right)^{2}\right] d x .
$$

Now, substituting Eq. (30) into Eq. (29), one obtains the equation of motion for the nanotube under the longitudinal magnetic field based on the NSGT

$$
\begin{aligned}
& -E I\left(1-l^{2} \frac{\partial^{2}}{\partial x^{2}}\right) \frac{\partial^{4} w}{\partial x^{4}}+\left\{\frac{E A}{2 L} \int_{0}^{L}\left(\frac{\partial w}{\partial x}\right)^{2} d x-\frac{E A}{L} l^{2} \int_{0}^{L}\left[\frac{\partial w}{\partial x} \frac{\partial^{3} w}{\partial x^{3}}+\left(\frac{\partial^{2} w}{\partial x^{2}}\right)^{2}\right] d x\right\}\left[\frac{\partial^{2} w}{\partial x^{2}}-(e a)^{2} \frac{\partial^{4} w}{\partial x^{4}}\right] \\
& -\rho A \frac{\partial^{2}}{\partial t^{2}}\left[w-(e a)^{2} \frac{\partial^{2} w}{\partial x^{2}}\right]-k_{w}\left[w-(e a)^{2} \frac{\partial^{2} w}{\partial x^{2}}\right]+\eta A H_{x}^{2} \frac{\partial^{2}}{\partial x^{2}}\left[w-(e a)^{2} \frac{\partial^{2} w}{\partial x^{2}}\right]=0 .
\end{aligned}
$$

Eq. (31) shows that the motion of the nanotube is governed by the nonlinear partial differential equation. This equation satisfies the following kinematic boundary conditions:

+ For the pinned-pinned (P-P) nanotube

$$
w(0, t)=w(L, t)=\frac{\partial^{2} w(0, t)}{\partial x^{2}}=\frac{\partial^{2} w(L, t)}{\partial x^{2}}=0
$$

+ For the clamped-clamped $(\mathrm{C}-\mathrm{C})$ nanotube

$$
w(0, t)=w(L, t)=\frac{\partial w(0, t)}{\partial x}=\frac{\partial w(L, t)}{\partial x}=0
$$

For convenience, the following dimensionless variables are defined

$$
\begin{aligned}
& \bar{x}=\frac{x}{L}, \bar{w}=\frac{w}{L}, \alpha=\frac{e a}{L}, \beta=\frac{l}{L}, \bar{t}=t \sqrt{\frac{E I}{\rho A L^{4}}}, \\
& \delta=\frac{L}{D}, h=\frac{d}{D}, \Gamma=\frac{\delta^{2}}{1+h^{2}}, K_{W}=\frac{k_{w} L^{4}}{E I}, H=\frac{\eta A H_{x}^{2} L^{2}}{E I} .
\end{aligned}
$$

Using Eq. (34), the equation of motion for the nanotube (31) is rewritten in the dimensionless form

$$
\begin{aligned}
& \beta^{2} \frac{\partial^{6} \bar{w}}{\partial \bar{x}^{6}}-\frac{\partial^{4} \bar{w}}{\partial \bar{x}^{4}}+16 \Gamma\left[\frac{1}{2} \int_{0}^{1}\left(\frac{\partial \bar{w}}{\partial \bar{x}}\right)^{2} d \bar{x}\right]\left(\frac{\partial^{2} \bar{w}}{\partial \bar{x}^{2}}-\alpha^{2} \frac{\partial^{4} \bar{w}}{\partial \bar{x}^{4}}\right)-16 \Gamma \beta^{2}\left[\int_{0}^{1}\left(\frac{\partial \bar{w}}{\partial \bar{x}} \frac{\partial^{3} \bar{w}}{\partial \bar{x}^{3}}\right) d \bar{x}\right]\left(\frac{\partial^{2} \bar{w}}{\partial \bar{x}^{2}}-\alpha^{2} \frac{\partial^{4} \bar{w}}{\partial \bar{x}^{4}}\right) \\
& -16 \Gamma \beta^{2}\left[\int_{0}^{1}\left(\frac{\partial^{2} \bar{w}}{\partial \bar{x}^{2}}\right)^{2} d \bar{x}\right]\left(\frac{\partial^{2} \bar{w}}{\partial \bar{x}^{2}}-\alpha^{2} \frac{\partial^{4} \bar{w}}{\partial \bar{x}^{4}}\right)-K_{W}\left(\bar{w}-\alpha^{2} \frac{\partial^{2} \bar{w}}{\partial \bar{x}^{2}}\right)+H\left(\frac{\partial^{2} \bar{w}}{\partial \bar{x}^{2}}-\alpha^{2} \frac{\partial^{4} \bar{w}}{\partial \bar{x}^{4}}\right)+\left(\alpha^{2} \frac{\partial^{4} \bar{w}}{\partial \bar{x}^{2} \partial \bar{t}^{2}}-\frac{\partial^{2} \bar{w}}{\partial \bar{t}^{2}}\right)=0 .
\end{aligned}
$$


Therefore, the kinematic boundary conditions (32) and (33) become:

+ For the pinned-pinned nanotube

$$
\bar{w}(0, \bar{t})=\bar{w}(L, \bar{t})=\frac{\partial^{2} \bar{w}(0, \bar{t})}{\partial \bar{x}^{2}}=\frac{\partial^{2} \bar{w}(L, \bar{t})}{\partial \bar{x}^{2}}=0 .
$$

+ For the clamped-clamped nanotube

$$
\bar{w}(0, \bar{t})=\bar{w}(L, \bar{t})=\frac{\partial \bar{w}(0, \bar{t})}{\partial \bar{x}}=\frac{\partial \bar{w}(L, \bar{t})}{\partial \bar{x}}=0 .
$$

It is very difficult to find the exact solution of Eq. (35), the approximate method is an effective tool to find the solution of this equation. First, the Galerkin technique is used to convert equation (35) into the ordinary differential one. To apply the Galerkin technique, the solution of Eq. (35), $\bar{w}(\bar{x}, \bar{t})$, is assumed to have the following form

$$
\bar{w}(\bar{x}, \bar{t})=Q(\bar{t}) \cdot \phi(\bar{x}),
$$

where $Q(\bar{t})$ is the time-dependent function must be determined and $\phi(\bar{x})$ is the shape function satisfying kinematic boundary conditions of the nanotube. The shape functions, $\phi(\bar{x})$, for pinned-pinned and clamped-clamped nanotubes can be chosen as in Tab. 1.

Table 1. The shape functions for Pinned-Pinned and Clamped-Clamped nanotubes

\begin{tabular}{cc}
\hline Boundary condition & Shape function \\
\hline Pinned-Pinned & $\phi(\bar{x})=\sin \pi \bar{x}$ \\
Clamped-Clamped & $\phi(\bar{x})=\frac{1}{2}(1-\cos 2 \pi \bar{x})$ \\
\hline
\end{tabular}

Applying the Galerkin technique, Eq. (35) is reduced to the following nonlinear ordinary differential equation

$$
\ddot{Q}(\bar{t})+\gamma_{1} Q(\bar{t})+\gamma_{2} Q^{3}(\bar{t})=0,
$$

where the coefficients $\gamma_{1}$ and $\gamma_{2}$ are determined by

$$
\gamma_{1}=\frac{\left\{\beta^{2} \int_{0}^{1} \varphi^{(6)} \varphi d \bar{x}-\int_{0}^{1} \varphi^{(4)} \varphi d \bar{x}-K_{W}\left[\int_{0}^{1} \varphi^{2} d \bar{x}-\alpha^{2} \int_{0}^{1} \varphi^{(2)} \varphi d \bar{x}\right]+H\left[\int_{0}^{1} \varphi^{(2)} \varphi d \bar{x}-\alpha^{2} \int_{0}^{1} \varphi^{(4)} \varphi d \bar{x}\right]\right\}}{\alpha^{2} \int_{0}^{1} \varphi^{(2)} \varphi d \bar{x}-\int_{0}^{1} \varphi^{2} d \bar{x}},
$$

$$
\gamma_{2}=\frac{16 \Gamma\left[\frac{1}{2} \int_{0}^{1}\left(\varphi^{\prime}\right)^{2} d \bar{x}-\beta^{2} \int_{0}^{1} \varphi^{\prime} \varphi^{(3)} d \bar{x}-\beta^{2} \int_{0}^{1}\left(\varphi^{(2)}\right)^{2} d \bar{x}\right]\left[\int_{0}^{1} \varphi^{(2)} \varphi d \bar{x}-\alpha^{2} \int_{0}^{1} \varphi^{(4)} \varphi d \bar{x}\right]}{\alpha^{2} \int_{0}^{1} \varphi^{(2)} \varphi d \bar{x}-\int_{0}^{1} \varphi^{2} d \bar{x}} .
$$


The nanotube is assumed to satisfy the following initial conditions

$$
Q(0)=Q_{0}, \dot{Q}(0)=0 \text {. }
$$

where $Q_{0}=\bar{w}_{\max }(0.5)$ is the dimensionless maximum vibration amplitude of the nanotube.

\section{SOLUTION PROCEDURE}

In this section, one will find the approximate solution of Eq. (39) with the initial conditions (42). It can see that Eq. (39) is cubic Duffing equation, there are many approximate methods to solve this equation [51]. In this work, the equivalent linearization method with the weighted averaging value [43-49] is employed to find the approximate solution of Eq. (39).

First, the equivalent linear form of the nonlinear equation (39) is introduced in the following form

$$
\ddot{Q}(\bar{t})+\omega^{2} Q(\bar{t})=0,
$$

where $\omega$ is known as the approximate frequency of the nanotube, which can be determined by the mean square error criterion

$$
\left\langle e^{2}(Q)\right\rangle=\left\langle\left(\gamma_{1} Q+\gamma_{2} Q^{3}-\omega^{2} Q\right)^{2}\right\rangle \rightarrow \min _{\omega^{2}}
$$

Thus, from the condition $\frac{\partial\left\langle e^{2}(Q)\right\rangle}{\partial \omega^{2}}=0$, leads to

$$
\omega=\sqrt{\gamma_{1}+\gamma_{2} \frac{\left\langle Q^{4}\right\rangle}{\left\langle Q^{2}\right\rangle}} .
$$

In Eq. (45), the symbol $\langle\cdot\rangle$ denotes the time-averaging operator which can be calculated using the definition of the weighted averaging value [43]

$$
\langle f(t)\rangle=\int_{0}^{+\infty} h(t) f(t) d t,
$$

with $h(t)$ is the weighted coefficient function which satisfies the following condition

$$
\int_{0}^{+\infty} h(t) d t=1 .
$$

In this work, a specific form of the weighted coefficient function is used [43]

$$
h(t)=s^{2} \omega t e^{-s \omega t}, s>0 .
$$

The solution of the linearized equation (43) has a form

$$
Q(\bar{t})=Q_{0} \cos (\omega \bar{t})
$$


With the periodic solution of linearized equation (43) given in Eq. (49), the averaging values $\left\langle Q^{2}\right\rangle$ and $\left\langle Q^{4}\right\rangle$ in Eq. (45) can be calculated by using Eq. (46) with the weighted coefficient function given in Eq. (48) and Laplace transform as follows

$$
\begin{gathered}
\left\langle Q^{2}\right\rangle=\left\langle Q_{0}^{2} \cos ^{2}(\omega t)\right\rangle=\int_{0}^{+\infty} Q_{0}^{2} s^{2} \omega \bar{t} e^{-s \omega \bar{t}} \cos ^{2}(\omega \bar{t}) d \bar{t} \\
=\int_{0}^{+\infty} Q_{0}^{2} s^{2} \tau e^{-s \tau} \cos ^{2}(\tau) d \tau=Q_{0}^{2} \frac{s^{4}+2 s^{2}+8}{\left(s^{2}+4\right)^{2}} . \\
\left\langle Q^{4}\right\rangle=\left\langle Q_{0}^{4} \cos ^{4}(\omega t)\right\rangle_{w}=\int_{0}^{+\infty} Q_{0}^{4} s^{2} \omega \bar{t} e^{-s \omega \bar{t}} \cos ^{4}(\omega \bar{t}) d \bar{t} \\
=\int_{0}^{+\infty} Q_{0}^{4} s^{2} \tau e^{-s \tau} \cos ^{4}(\tau) d \tau=Q_{0}^{4} \frac{s^{8}+28 s^{6}+248 s^{4}+416 s^{2}+1536}{\left(s^{2}+4\right)^{2}\left(s^{2}+16\right)^{2}} .
\end{gathered}
$$

Substituting the averaging values in Eqs. (50) and (51) into Eq. (45), the approximate frequency can be get as

$$
\omega_{N L}=\sqrt{\gamma_{1}+\gamma_{2} \frac{248 s^{4}+416 s^{2}+1536+28 s^{6}+s^{8}}{\left(s^{4}+2 s^{2}+8\right)\left(s^{2}+16\right)^{2}} Q_{0}^{2}}
$$

It can be seen that the approximate frequency of the nanotube depends not only on the initial amplitude $Q_{0}$ but also on the parameter $s$. With $s=2$, the obtained results show the accuracy [44-49]. Thus, when $s=2$, one obtains

$$
\omega_{N L}=\sqrt{\gamma_{1}+0.72 \gamma_{2} Q_{0}^{2}}
$$

The approximate solution of Eq. (39) can be get as follows

$$
Q(\bar{t})=\alpha \cos \left(\sqrt{\gamma_{1}+0.72 \gamma_{2} Q_{0}^{2}} \bar{t}\right) .
$$

By using the shape functions in Tab. 1 and integrals in Eqs. (40) and (41), one obtains the approximate nonlinear frequencies of the nanotubes:

+ For pinned-pinned nanotube

$$
\omega_{N L}=\sqrt{\pi^{4} \frac{1+\beta^{2} \pi^{2}}{1+\alpha^{2} \pi^{2}}+K_{W}+H \pi^{2}+2.88 \Gamma \pi^{4} Q_{0}^{2}} .
$$

+ For clamped-clamped nanotube

$$
\omega_{N L}=\sqrt{16 \pi^{4} \frac{1+4 \beta^{2} \pi^{2}}{3+4 \alpha^{2} \pi^{2}}+K_{W}+4 H \pi^{2} \frac{1+4 \alpha^{2} \pi^{2}}{3+4 \alpha^{2} \pi^{2}}+11.52 \Gamma \pi^{4} \frac{1+4 \alpha^{2} \pi^{2}}{3+4 \alpha^{2} \pi^{2}} Q_{0}^{2}} .
$$




\section{RESULTS AND DISCUSSIONS}

It can be seen from Eqs. (55) and (56) that the material length scale parameter $(\beta=$ $l / L)$, the Winkler parameter $\left(K_{W}\right)$, the magnetic field $(H)$, the aspect ratio $(\delta=L / D)$ and the initial amplitude $\left(Q_{0}\right)$ lead to an increase of the nonlinear frequency $\left(\omega_{N L}\right)$ of the nanotubes, while the nonlocal parameter $(\alpha=e a / L)$ and the diameter ratio $(h=d / D)$ lead to a decrease of the nonlinear frequency $\left(\omega_{N L}\right)$ of the nanotubes. To study nonlinear vibration responses of the nanotubes, one introduces the scale ratio

$$
c=\frac{\beta}{\alpha}=\frac{l}{e a},
$$

and the frequency ratio (the ratio of the nonlinear frequency to the linear frequency)

$$
\omega_{\text {ratio }}=\frac{\omega_{N L}}{\omega_{L}} .
$$

Note that the linear frequency $\omega_{L}$ can be achieved from the nonlinear one by letting $Q_{0}=0$.

\subsection{Validation of model}

The exact frequency of Eq. (39) is given as [51]

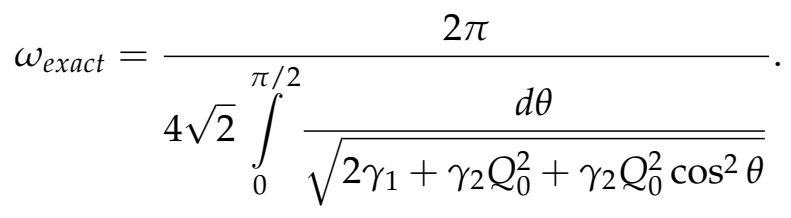

Comparison of the approximate frequency with the exact frequency for $K_{W}=10$, $H=20, L / D=20, d / D=0.8$ and several different values of the initial amplitude $Q_{0}$, the material length scale parameter $\beta$ and the nonlocal parameter $\alpha$ is shown in Tabs. 2

Table 2. Comparison of the approximate frequency with the exact frequency for P-P nanotube

\begin{tabular}{ccccc}
\hline$Q_{0}$ & $\beta$ & $\alpha$ & $\omega_{\text {present }}$ & $\omega_{\text {exact }}$ \\
\hline \multirow{3}{*}{0.01} & 0.1 & 0.1 & 17.6615 & 17.6613 \\
\cline { 2 - 5 } & 0.2 & 0.1 & 18.3896 & 18.3894 \\
\cline { 2 - 5 } & 0.1 & 0.2 & 17.0660 & 17.0658 \\
\hline \multirow{2}{*}{0.05} & 0.1 & 0.1 & 21.9770 & 21.9143 \\
\cline { 2 - 5 } & 0.2 & 0.1 & 22.5663 & 22.5084 \\
\hline \multirow{2}{*}{0.1} & 0.1 & 0.2 & 21.5014 & 21.4344 \\
& 0.1 & 0.1 & 31.8990 & 31.5664 \\
& 0.2 & 0.1 & 32.3979 & 32.9880 \\
\hline
\end{tabular}


and 3 corresponding to P-P nanotube and C-C nanotube, respectively. The accuracy of analytical solutions can be observed from these tables.

Table 3. Comparison of the approximate frequency with the exact frequency for C-C nanotube

\begin{tabular}{ccccc}
\hline$Q_{0}$ & $\beta$ & $\alpha$ & $\omega_{\text {present }}$ & $\omega_{\text {exact }}$ \\
\hline \multirow{3}{*}{0.01} & 0.1 & 0.1 & 31.4079 & 31.4078 \\
\cline { 2 - 5 } & 0.2 & 0.1 & 39.1177 & 39.1176 \\
\cline { 2 - 5 } & 0.1 & 0.2 & 30.7489 & 30.7487 \\
\hline \multirow{3}{*}{0.05} & 0.1 & 0.1 & 35.6032 & 35.5635 \\
& 0.2 & 0.1 & 42.5596 & 42.5364 \\
\hline \multirow{2}{*}{0.1} & 0.1 & 0.2 & 36.4813 & 36.4119 \\
\cline { 2 - 5 } & 0.1 & 0.1 & 46.3261 & 46.0351 \\
\hline & 0.2 & 0.1 & 51.8637 & 51.6572 \\
\hline
\end{tabular}

Based on the nonlocal elasticity theory, Chang [52] examined the nonlinear vibration of nanobeams under magnetic field. The frequency ratios of the nanobeams obtained in this work and those obtained by Chang [52] are compared and shown in Tab. 4. Note that the results for the nanobeams can be get from the obtained results for nanotubes by letting the inner diameter equal to zero $(d=0)$. A good agreement can be seen between the frequency ratios obtained in this work and the frequency ratios obtained by Chang [52].

Table 4. Comparison of the frequency ratios of nanobeams with $H=50$ and $L / h=20$

\begin{tabular}{cccccc}
\hline \multirow{2}{*}{$Q_{0}$} & \multirow{2}{*}{$\alpha$} & \multicolumn{2}{c}{ P-P nanobeam } & \multicolumn{2}{c}{ C-C nanobeam } \\
\cline { 2 - 6 } & & Chang [52] & Present & Chang [52] & Present \\
\hline \multirow{3}{*}{0.01} & 0 & 1.0098 & 1.0095 & 1.0066 & 1.0063 \\
\cline { 2 - 6 } & 0.1 & 1.0100 & 1.0096 & 1.0075 & 1.0072 \\
\cline { 2 - 6 } & 0.2 & 1.0103 & 1.0099 & 1.0090 & 1.0087 \\
\cline { 2 - 6 } & 0.3 & 1.0107 & 1.0102 & 1.0100 & 1.0096 \\
\hline \multirow{3}{*}{0.1} & 0 & 1.7258 & 1.7027 & 1.5243 & 1.5069 \\
\cline { 2 - 6 } & 0.1 & 1.7343 & 1.7110 & 1.5851 & 1.5659 \\
\cline { 2 - 6 } & 0.2 & 1.7536 & 1.7298 & 1.6774 & 1.6556 \\
\hline & 0.3 & 1.7733 & 1.7489 & 1.7374 & 1.7140 \\
\hline
\end{tabular}


Furthermore, for P-P nonlocal nanotube $(\beta=0)$ and without the magnetic field $(H=0)$, the linear frequency derived from Eq. (45) is the same as the linear frequency achieved by Wang and Li [18].

\subsection{Effects of the nonlocal parameter and the material length scale parameter}

Effects of the nonlocal and material length scale parameters on the nonlinear vibration response of the nanotubes are shown in Figs. 2-4. Fig. 2 presents the variation of the frequency ratios $\omega_{\text {ratio }}$ of the nanotubes to the scale ratio $c$ for $L / D=20, d / D=0.8$, $K_{W}=50, H=50, Q_{0}=0.1$ and some different values of the nonlocal parameter. And for case of $L / D=20, d / D=0.8, K_{W}=50, H=50, Q_{0}=0.1$ and some different values of the scale ratio, the variations of the frequency ratios to the nonlocal parameter and the material length scale parameter are plotted in Figs. 3 and 4 , respectively. It can be seen that the frequency ratios of the classical nanotubes $(e a / L=0, c=0)$ are equal to the frequency ratios of the SGT nanotubes with $c=1$. From Fig. 2, it can see that the frequency ratios decrease when the scale ratio increases. When $c<1$ (i.e., $l<e a$ ), the frequency ratios of the nanotube increase as the nonlocal parameter $e a / L$ increases. However, when $c>1$ (i.e., $l>e a$ ), the frequency ratios of the nanotube decrease as the nonlocal parameter $e a / L$ increases. Fig. 3 reveals that the frequency ratios of the NSGT nanotubes are always smaller than the ones of the nonlocal nanotubes $(c=0)$. From Figs. 3 and 4, it can observe that when $c<1$, the frequency ratios of the nanotubes increase as the nonlocal and length scale parameters increase; while $c>1$, the frequency ratios of the nanotubes decrease as the nonlocal and length scale parameters increase. It means that the nanotubes exert a softening effect when $c<1$, and exert a hardening effect when $c>1$.

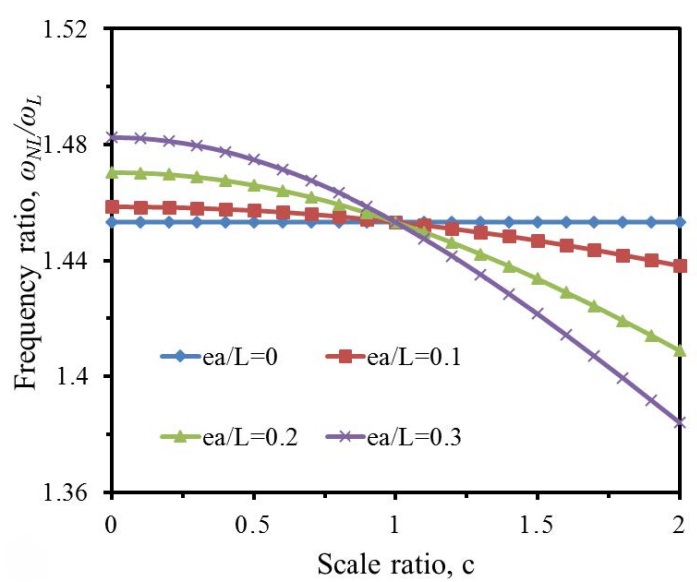

(a) P-P nanotube

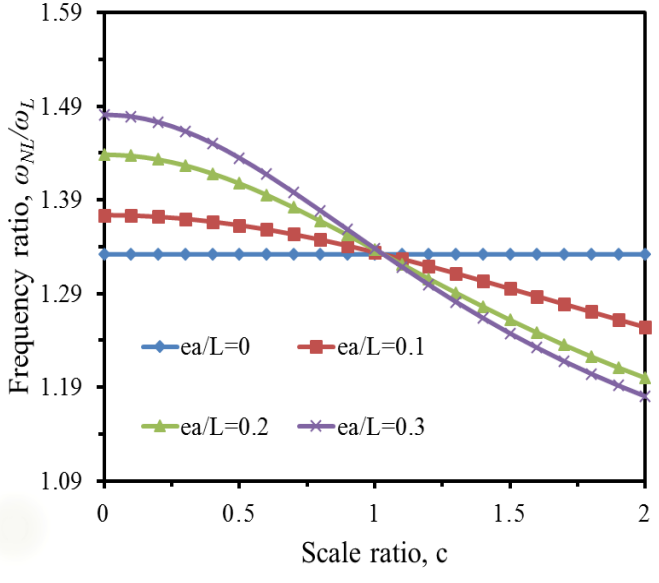

(b) C-C nanotube

Fig. 2. The variation of the frequency ratios to the scale ratio for some values of the nonlocal parameter 


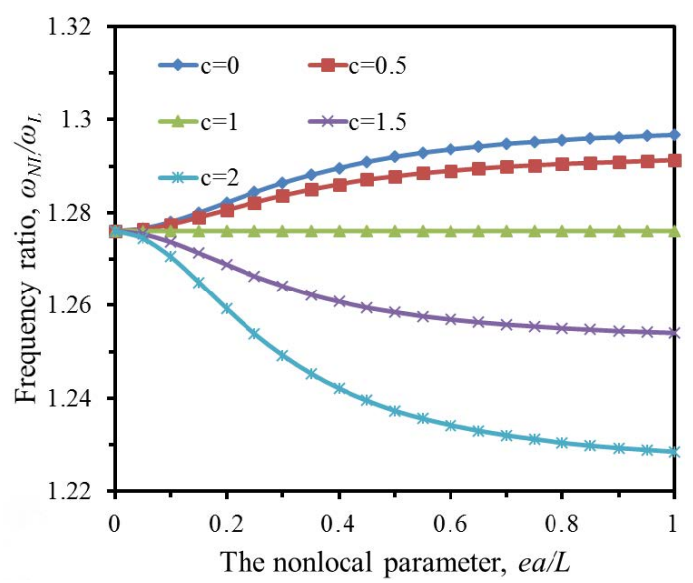

(a) P-P nanotube

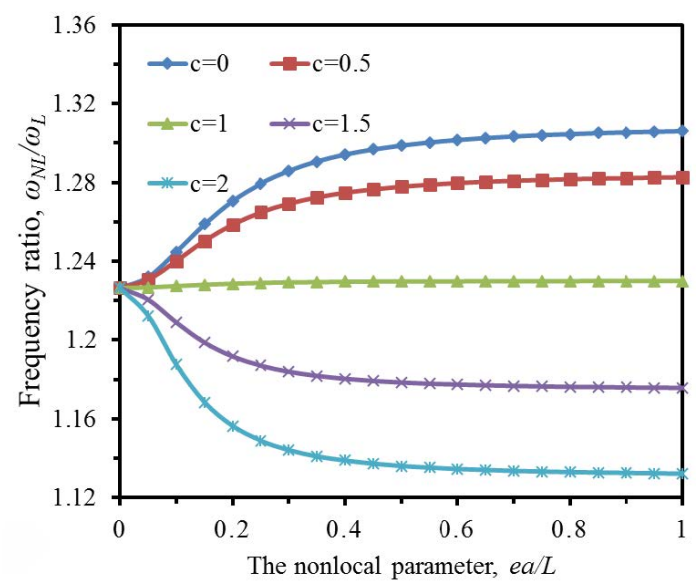

(b) C-C nanotube

Fig. 3. The variation of the frequency ratios to the nonlocal parameter for some values of the scale ratio

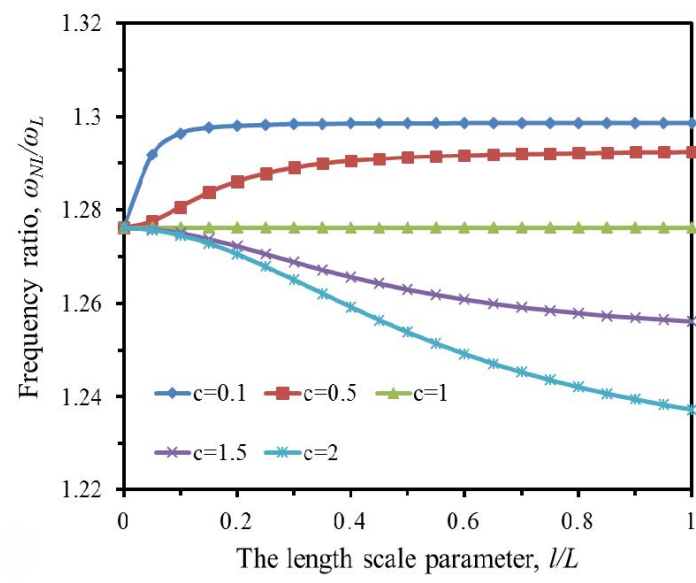

(a) P-P nanotube

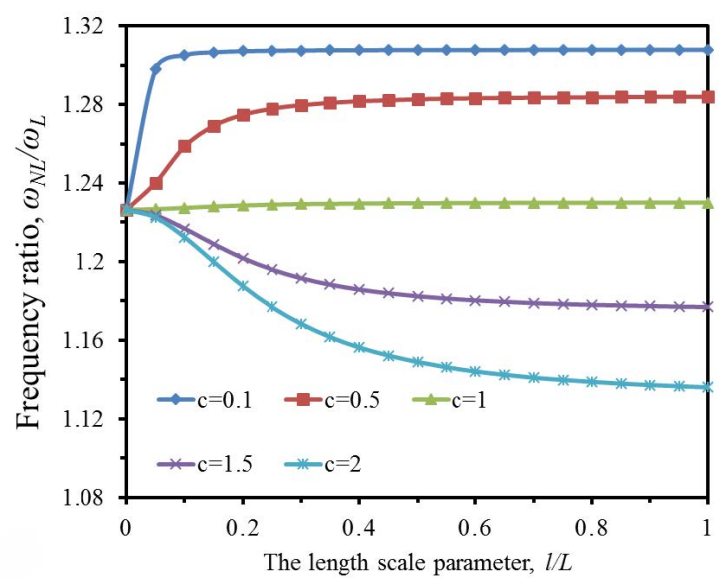

(b) C-C nanotube

Fig. 4. The variation of the frequency ratios to the material length scale parameter for some values of the scale ratio

\subsection{Effect of the diameter ratio}

For case of $\alpha=0.2, L / D=20, K_{W}=50, H=50, Q_{0}=0.1$ and some values of the diameter ratio $(d / D)$, the variation of the frequency ratios of the nanotubes to the scale ratio is presented in Fig. 5. And Fig. 6 shows the variation of the frequency ratios of the nanotubes to the diameter ratio for $\alpha=0.2, L / D=20, K_{W}=50, H=50, Q_{0}=0.1$ and some different values of the scale ratio. As can see from Fig. 5 that the frequency ratios 
of the nanotubes are always smaller than the ones of the nanobeams $(d / D=0)$; and the frequency ratios of the nanotubes decrease when the diameter ratio increases. From Fig. 6, it can see that the frequency ratios of the NSGT nanotubes are always smaller than the ones of the nonlocal nanotubes ( $c=0$ i.e., $l=0)$; and for the NSGT, the scale ratio leads to a decrease in the frequency ratios of the nanotubes.

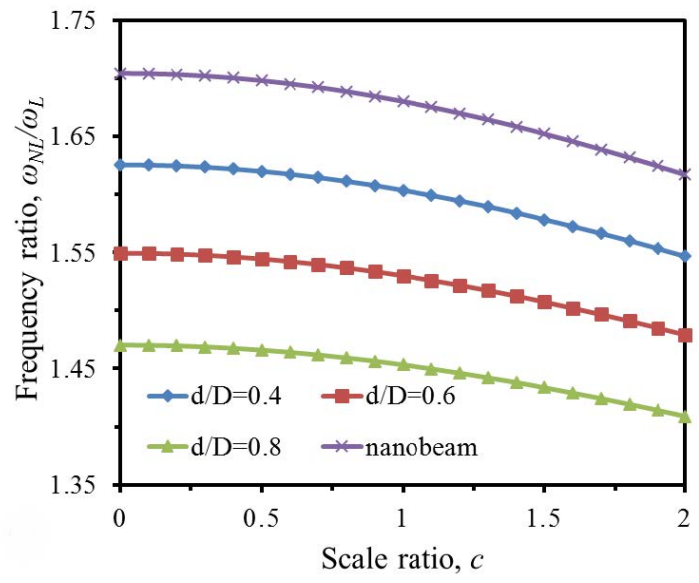

(a) P-P nanotube

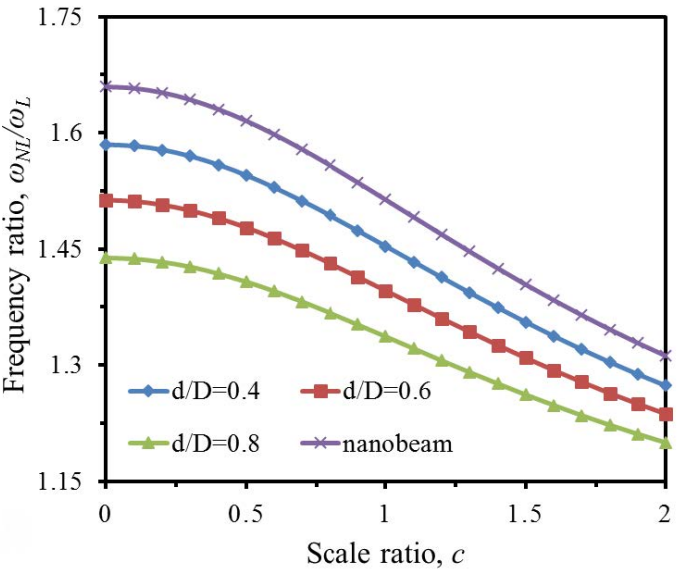

(b) C-C nanotube

Fig. 5. The variation of the frequency ratios to the scale ratio for some values of the diameter ratio

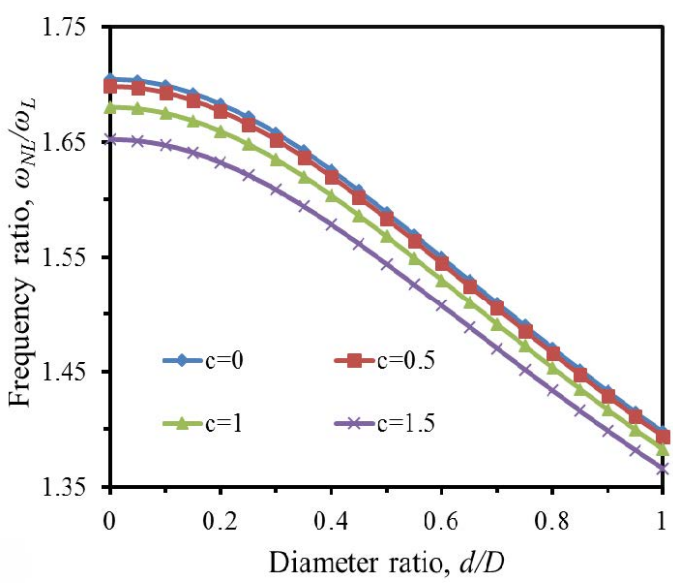

(a) P-P nanotube

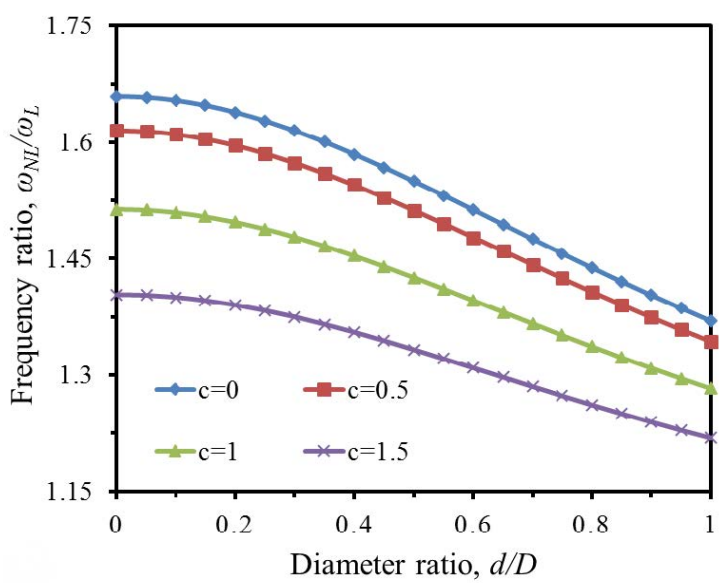

(b) C-C nanotube

Fig. 6. The variation of the frequency ratios to the diameter ratio for some values of the scale parameter 


\subsection{Effect of the aspect ratio}

The aspect ratio $L / D$ has an important influence on the nonlinear vibration responses of the nanotubes as shown in Figs. 7 and 8. For $\alpha=0.3, d / D=0.8, K_{W}=20, H=50$ and $Q_{0}=0.1$, the variations of the frequency ratios of the nanotubes to the scale ratio and the aspect ratio are plotted in Figs. 7 and 8 corresponding to several different values of the aspect ratio and the scale ratio, respectively. It can be concluded that the frequency

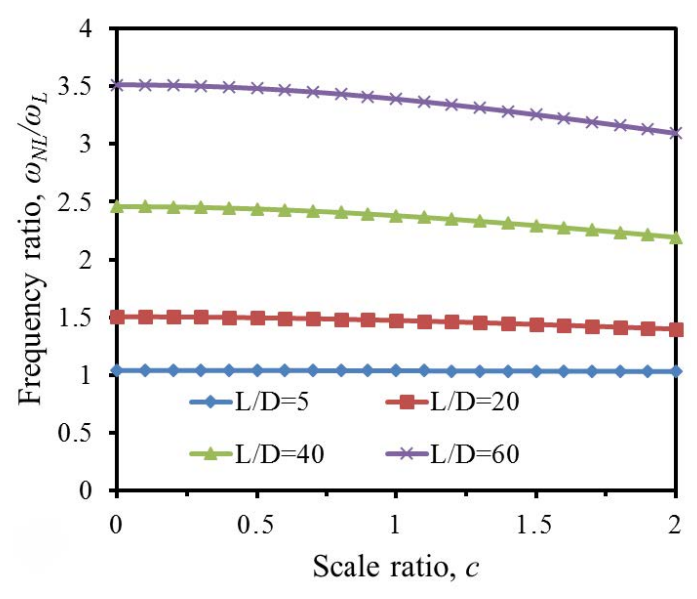

(a) P-P nanotube

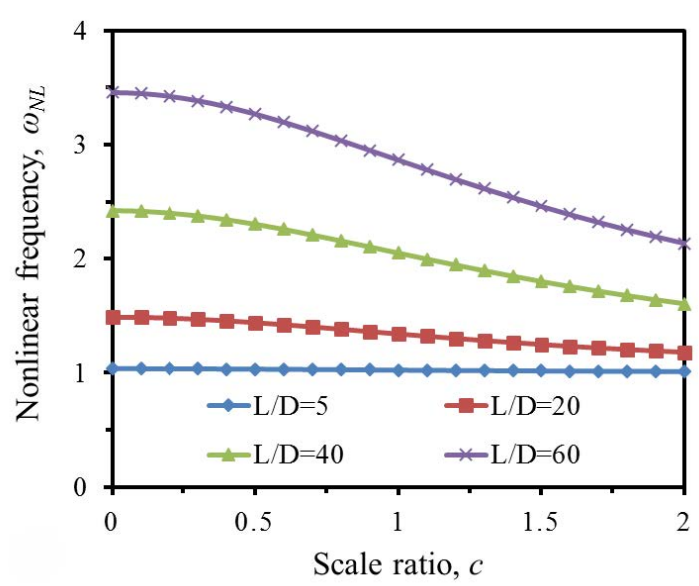

(b) C-C nanotube

Fig. 7. The variation of the frequency ratios to the scale ratio for some values of the aspect ratio

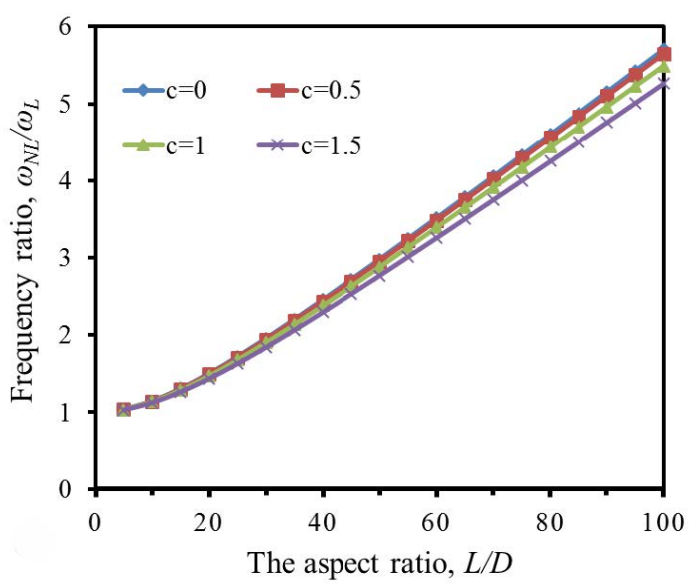

(a) P-P nanotube

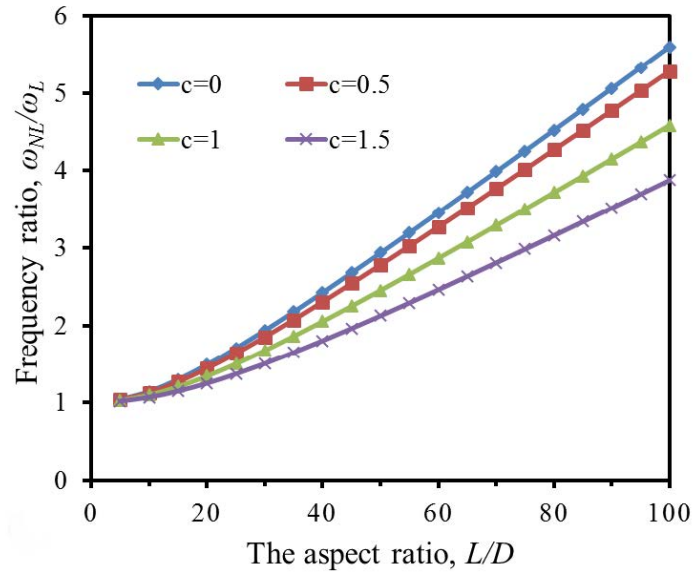

(b) C-C nanotube

Fig. 8. The variation of the frequency ratios to the aspect ratio for some values of the scale ratio 
ratios of the nanotubes increases as the aspect ratio increases. And for a fixed value of the aspect ratio, the frequency ratios of the nanotubes decrease as the scale ratio increases. However, the frequency ratios of the NSGT nanotubes are always smaller than the ones of the nonlocal nanotubes $(c=0)$.

\subsection{Effect of the elastic foundation}

Figs. 9 and 10 show effect of the Winkler parameter $K_{W}$ on the frequency ratios of the nanotubes. Fig. 9 presents the variation of the frequency ratios of the nanotubes to

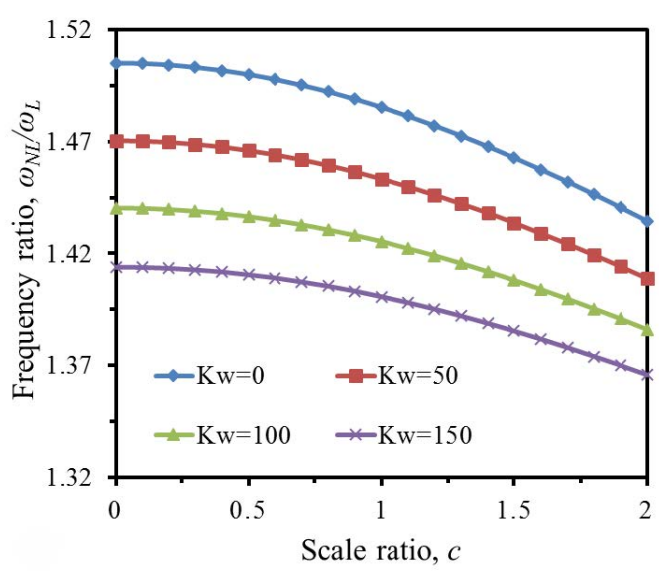

(a) P-P nanotube

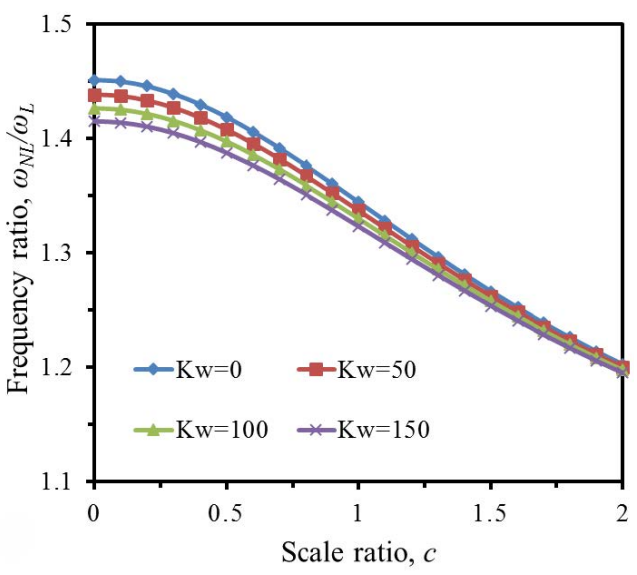

(b) C-C nanotube

Fig. 9. The variation of the frequency ratios to the scale ratio for some values of the Winkler parameter

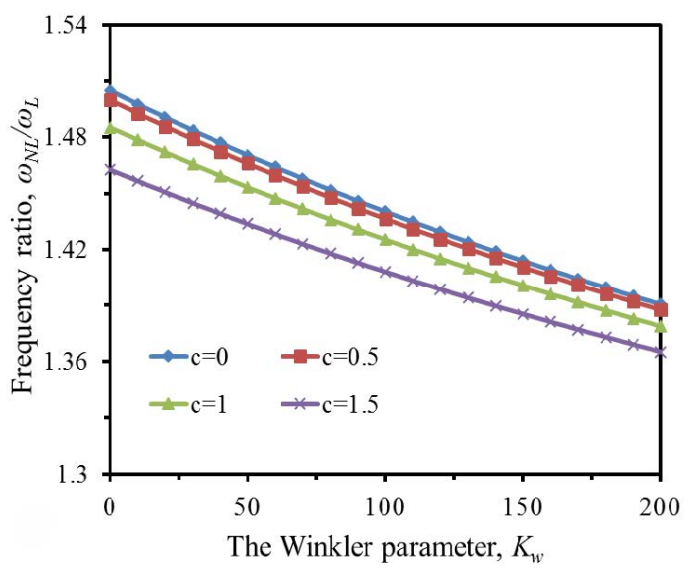

(a) P-P nanotube

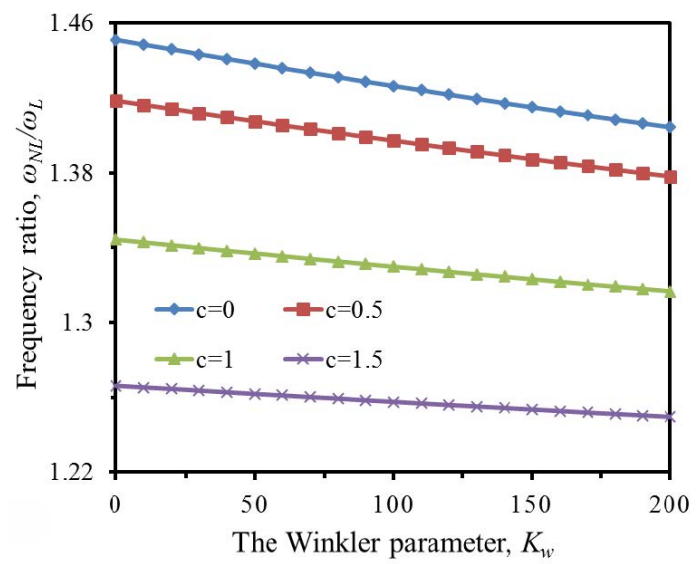

(b) C-C nanotube

Fig. 10. The variation of the frequency ratios to the Winkler parameter for some values of the scale ratio 
the scale ratio for $\alpha=0.2, d / D=0.8, L / D=20, H=50, Q_{0}=0.1$ and some values of the Winkler parameter; while Fig. 10 shows the variation of the frequency ratios of the nanotubes to the Winkler parameter for $\alpha=0.2, d / D=0.8, L / D=20, H=50, Q_{0}=0.1$ and some values of the scale ratio. As can see from these Figures that the Winkler parameter leads to a decrease of the frequency ratios of the nanotubes. The fact that the elastic foundation enhances the stiffness of the nanotubes, so the frequency of the nanotubes increases by increasing the coefficient of the elastic foundation. However, the increase in the nonlinear frequency due to the elastic foundation coefficient is smaller than the increase in the linear frequency, thus the frequency ratios of the nanotubes decreases when the elastic foundation coefficient increases.

\subsection{Effect of the magnetic field}

Final, effect of the magnetic field on the frequency ratios of the nanotubes is investigated in this sub-section. The variation of the frequency ratios of the nanotubes to the scale ratio for $\alpha=0.2, d / D=0.8, L / D=20, K_{W}=30, Q_{0}=0.1$ and some values of the magnetic field is presented in Fig. 11. And Fig. 12 shows the variation of the frequency ratios of the nanotubes to the magnetic field for $\alpha=0.2, d / D=0.8, L / D=20$, $K_{W}=30, Q_{0}=0.1$ and some values of the scale ratio. It can be concluded that effect of the magnetic field has the same as effect of the elastic foundation to the nonlinear vibration responses of the nanotubes. An increase in the magnetic field leads to a decrease of the frequency ratios of the nanotubes, this observation is completely consistent with the results obtained by Chang [52]. And for a fixed value of the magnetic field, the frequency ratios of the nanotubes decrease as the scale ratio increases. The magnetic field has an important influence on the nonlinear vibration responses of the nanotubes.

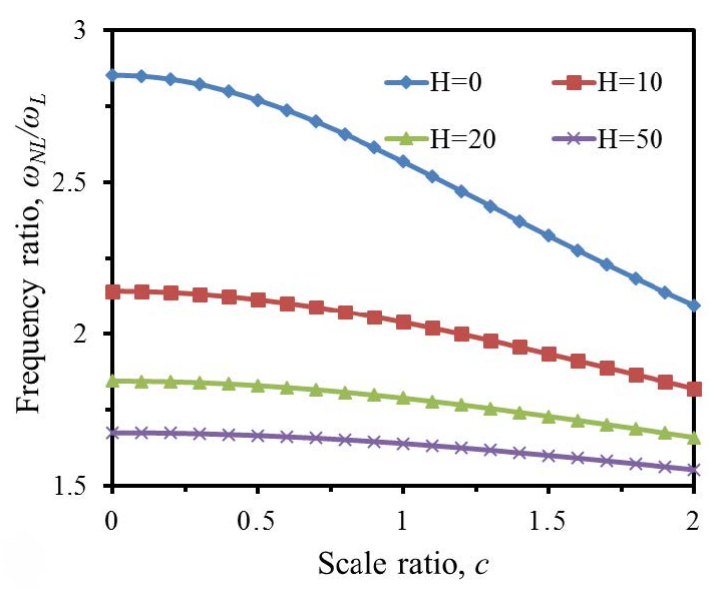

(a) P-P nanotube

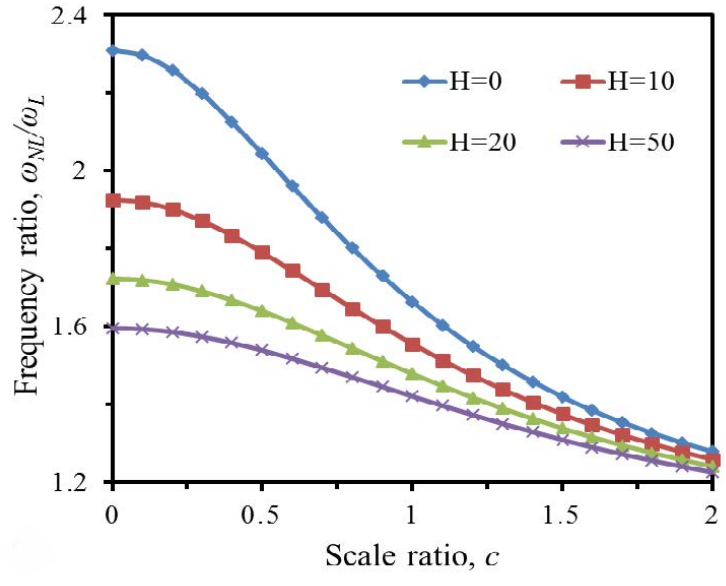

(b) C-C nanotube

Fig. 11. The variation of the frequency ratios to the scale ratio for some values of the magnetic field 


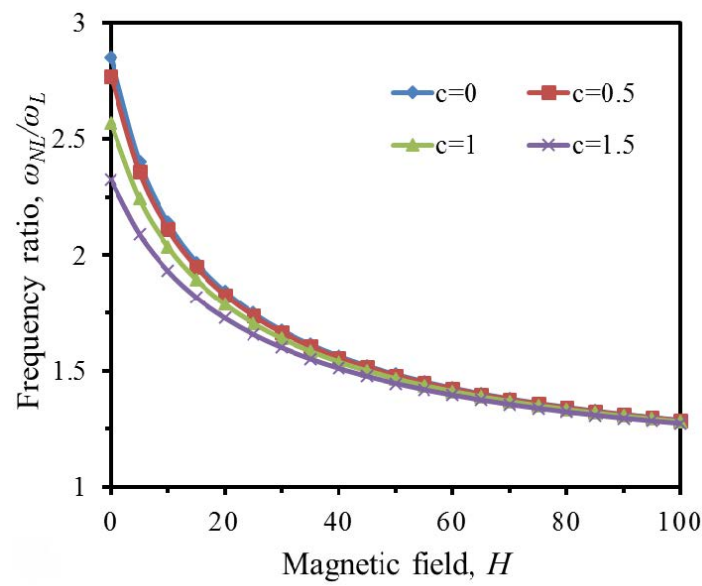

(a) P-P nanotube

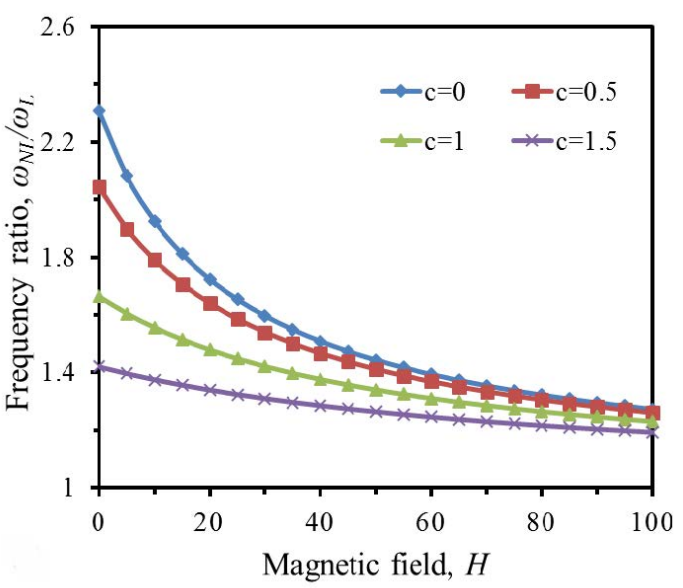

(b) C-C nanotube

Fig. 12. The variation of the frequency ratios to the magnetic field for some values of the scale ratio

\section{CONCLUSIONS}

The nonlinear vibration response of the nanotubes under the magnetic field based on the nonlocal strain gradient theory and the Euler-Bernoulli beam theory is investigated in this work. The equation of motion for the nanotubes is derived by the Hamilton principle. The Glerkin technique and the equivalent linearization with the weighted averaging value are employed to find the approximate frequencies of the nanotubes with pinnedpinned and clamped-clamped boundary conditions. The comparison of the obtained approximate solutions with the exact ones and the published ones shows the accuracy of the obtained approximate solutions.

Effects of the nonlocal parameter $e a / L$, the material length scale parameter $l / L$, the aspect ratio $L / D$, the diameter ratio $d / D$, the elastic foundation $K_{W}$ and the magnetic field $H$ on the nonlinear vibration responses of the nanotubes are examined. It can be concluded that:

- The nonlocal parameter leads to a decrease in the nonlinear frequencies of the nanotubes; while the material length scale parameter, the aspect ratio, the diameter ratio, the elastic foundation and the magnetic field lead to an increase in the nonlinear frequencies of the nanotubes.

- When $l<e a$, the frequency ratios of the nanotube increase as the nonlocal parameter $e a / L$ increases. However, when $l>e a$, the frequency ratios of the nanotube decrease as the nonlocal parameter $e a / L$ increases. The frequency ratios of the NSGT nanotubes are always smaller than the ones of the nonlocal nanotubes $(c=l / e a=0)$. When $c<1$, the frequency ratios of the nanotubes increase as the nonlocal and length scale parameters increase; while $c>1$, the frequency ratios of the nanotubes decrease as the nonlocal and length scale parameters increase. 
- The diameter ratio, the elastic foundation and the magnetic field lead to a decrease in the frequency ratios of the nanotubes; while the aspect ratio leads to an increase in the frequency ratios of the nanotubes.

\section{ACKNOWLEDGMENT}

This work was supported by Vietnam National Foundation for Science and Technology Development (NAFOSTED) under grant number 107.02-2020.03.

\section{REFERENCES}

[1] S. Iijima. Helical microtubules of graphitic carbon. Nature, 354, (6348), (1991), pp. 56-58. https://doi.org/10.1038/354056a0.

[2] S. Chakraverty and S. K. Jena. Free vibration of single walled carbon nanotube resting on exponentially varying elastic foundation. Curved and Layered Structures, 5, (1), (2018), pp. 260272. https://doi.org/10.1515/cls-2018-0019.

[3] P. Poncharal, Z. L. Wang, D. Ugarte, and W. A. De Heer. Electrostatic deflections and electromechanical resonances of carbon nanotubes. Science, 283, (5407), (1999), pp. 1513-1516. https://doi.org/10.1126/science.283.5407.1513.

[4] J. A. Pelesko and D. H. Bernstein. Modeling MEMS and NEMS. FL: Chapman \& Hall/CRC, Boca Raton, (2003).

[5] K. Tsukagoshi, N. Yoneya, S. Uryu, Y. Aoyagi, A. Kanda, Y. Ootuka, and B. W. Alphenaar. Carbon nanotube devices for nanoelectronics. Physica B: Condensed Matter, 323, (1-4), (2002), pp. 107-114. https://doi.org/10.1016/s0921-4526(02)00993-6.

[6] W. B. Choi, E. Bae, D. Kang, S. Chae, B.-h. Cheong, J.-h. Ko, E. Lee, and W. Park. Aligned carbon nanotubes for nanoelectronics. Nanotechnology, 15, (10), (2004). https://doi.org/10.1088/0957-4484/15/10/003.

[7] M. Samadishadlou, M. Farshbaf, N. Annabi, T. Kavetskyy, R. Khalilov, S. Saghfi, A. Akbarzadeh, and S. Mousavi. Magnetic carbon nanotubes: preparation, physical properties, and applications in biomedicine. Artificial Cells, Nanomedicine, and Biotechnology, 46, (7), (2018), pp. 1314-1330. https://doi.org/10.1080/21691401.2017.1389746.

[8] Y. Gao and Y. Bando. Carbon nanothermometer containing gallium. Nature, 415, (6872), (2002), pp. 599-599. https://doi.org/10.1038/415599a.

[9] G. Hummer, J. C. Rasaiah, and J. P. Noworyta. Water conduction through the hydrophobic channel of a carbon nanotube. Nature, 414, (6860), (2001), pp. 188-190. https://doi.org/10.1038/35102535.

[10] A. C. Eringen and D. G. B. Edelen. On nonlocal elasticity. International Journal of Engineering Science, 10, (3), (1972), pp. 233-248. https://doi.org/10.1016/0020-7225(72)90039-0.

[11] A. C. Eringen. On differential equations of nonlocal elasticity and solutions of screw dislocation and surface waves. Journal of Applied Physics, 54, (9), (1983), pp. 4703-4710. https://doi.org/10.1063/1.332803.

[12] R. D. Mindlin. Micro-structure in linear elasticity. Archive for Rational Mechanics and Analysis, 16, (1), (1964), pp. 51-78. https://doi.org/10.1007/bf00248490.

[13] R. D. Mindlin. Second gradient of strain and surface-tension in linear elasticity. International Journal of Solids and Structures, 1, (4), (1965), pp. 417-438. https://doi.org/10.1016/00207683(65)90006-5. 
[14] F. A. C. M. Yang, A. C. M. Chong, D. C. C. Lam, and P. Tong. Couple stress based strain gradient theory for elasticity. International Journal of Solids and Structures, 39, (10), (2002), pp. 27312743. https://doi.org/10.1016/s0020-7683(02)00152-x.

[15] J. Yang, L. L. Ke, and S. Kitipornchai. Nonlinear free vibration of single-walled carbon nanotubes using nonlocal Timoshenko beam theory. Physica E: Low-dimensional Systems and Nanostructures, 42, (5), (2010), pp. 1727-1735. https://doi.org/10.1016/j.physe.2010.01.035.

[16] S. Narendar, S. S. Gupta, and S. Gopalakrishnan. Wave propagation in singlewalled carbon nanotube under longitudinal magnetic field using nonlocal EulerBernoulli beam theory. Applied Mathematical Modelling, 36, (9), (2012), pp. 4529-4538. https://doi.org/10.1016/j.apm.2011.11.073.

[17] Z. Zhang, Y. Liu, and B. Li. Free vibration analysis of fluid-conveying carbon nanotube via wave method. Acta Mechanica Solida Sinica, 27, (6), (2014), pp. 626-634. https://doi.org/10.1016/s0894-9166(15)60007-6.

[18] Y.-Z. Wang and F.-M. Li. Nonlinear free vibration of nanotube with small scale effects embedded in viscous matrix. Mechanics Research Communications, 60, (2014), pp. 45-51. https://doi.org/10.1016/j.mechrescom.2014.06.002.

[19] Y.-X. Zhen and B. Fang. Nonlinear vibration of fluid-conveying single-walled carbon nanotubes under harmonic excitation. International Journal of Non-Linear Mechanics, 76, (2015), pp. 48-55. https://doi.org/10.1016/j.ijnonlinmec.2015.05.005.

[20] P. Valipour, S. E. Ghasemi, M. R. Khosravani, and D. D. Ganji. Theoretical analysis on nonlinear vibration of fluid flow in single-walled carbon nanotube. Journal of Theoretical and Applied Physics, 10, (3), (2016), pp. 211-218. https://doi.org/10.1007/s40094-016-0217-9.

[21] M. Sadeghi-Goughari, S. Jeon, and H.-J. Kwon. Effects of magnetic-fluid flow on structural instability of a carbon nanotube conveying nanoflow under a longitudinal magnetic field. Physics Letters A, 381, (35), (2017), pp. 2898-2905. https://doi.org/10.1016/j.physleta.2017.06.054.

[22] L. Wang. Size-dependent vibration characteristics of fluid-conveying microtubes. Journal of Fluids and Structures, 26, (4), (2010), pp. 675-684. https://doi.org/10.1016/j.jfluidstructs.2010.02.005.

[23] L. Wang, H. T. Liu, Q. Ni, and Y. Wu. Flexural vibrations of microscale pipes conveying fluid by considering the size effects of micro-flow and micro-structure. International Journal of Engineering Science, 71, (2013), pp. 92-101. https://doi.org/10.1016/j.ijengsci.2013.06.006.

[24] M. Tang, Q. Ni, L. Wang, Y. Luo, and Y. Wang. Nonlinear modeling and sizedependent vibration analysis of curved microtubes conveying fluid based on modified couple stress theory. International Journal of Engineering Science, 84, (2014), pp. 1-10. https://doi.org/10.1016/j.ijengsci.2014.06.007.

[25] W. Xia and L. Wang. Microfluid-induced vibration and stability of structures modeled as microscale pipes conveying fluid based on non-classical Timoshenko beam theory. Microfluidics and Nanofluidics, 9, (4-5), (2010), pp. 955-962. https://doi.org/10.1007/s10404-010-0618-z.

[26] M. R. Ghazavi, H. Molki, and A. A. Beigloo. Nonlinear analysis of the micro/nanotube conveying fluid based on second strain gradient theory. Applied Mathematical Modelling, 60, (2018), pp. 77-93. https://doi.org/10.1016/j.apm.2018.03.013.

[27] C. W. Lim, G. Zhang, and J. N. Reddy. A higher-order nonlocal elasticity and strain gradient theory and its applications in wave propagation. Journal of the Mechanics and Physics of Solids, 78, (2015), pp. 298-313. https://doi.org/10.1016/j.jmps.2015.02.001. 
[28] M. Şimşek. Nonlinear free vibration of a functionally graded nanobeam using nonlocal strain gradient theory and a novel Hamiltonian approach. International Journal of Engineering Science, 105, (2016), pp. 12-27. https://doi.org/10.1016/j.ijengsci.2016.04.013.

[29] M. N. M. Allam and A. F. Radwan. Nonlocal strain gradient theory for bending, buckling, and vibration of viscoelastic functionally graded curved nanobeam embedded in an elastic medium. Advances in Mechanical Engineering, 11, (4), (2019). https://doi.org/10.1177/1687814019837067.

[30] S. Esfahani, S. E. Khadem, and A. E. Mamaghani. Nonlinear vibration analysis of an electrostatic functionally graded nano-resonator with surface effects based on nonlocal strain gradient theory. International Journal of Mechanical Sciences, 151, (2019), pp. 508-522. https://doi.org/10.1016/j.ijmecsci.2018.11.030.

[31] V.-H. Dang, D.-A. Nguyen, M.-Q. Le, and T.-H. Duong. Nonlinear vibration of nanobeams under electrostatic force based on the nonlocal strain gradient theory. International Journal of Mechanics and Materials in Design, (2019), pp. 1-20. https://doi.org/10.1007/s10999-01909468-8.

[32] R. Bahaadini, A. R. Saidi, and M. Hosseini. Flow-induced vibration and stability analysis of carbon nanotubes based on the nonlocal strain gradient Timoshenko beam theory. Journal of Vibration and Control, 25, (1), (2019), pp. 203-218. https://doi.org/10.1177/1077546318774242.

[33] M. Malikan, V. B. Nguyen, and F. Tornabene. Damped forced vibration analysis of singlewalled carbon nanotubes resting on viscoelastic foundation in thermal environment using nonlocal strain gradient theory. Engineering Science and Technology, 21, (4), (2018), pp. 778786. https://doi.org/10.1016/j.jestch.2018.06.001.

[34] G.-L. She, Y.-R. Ren, F.-G. Yuan, and W.-S. Xiao. On vibrations of porous nanotubes. International Journal of Engineering Science, 125, (2018), pp. 23-35. https://doi.org/10.1016/j.ijengsci.2017.12.009.

[35] M. Atashafrooz, R. Bahaadini, and H. R. Sheibani. Nonlocal, strain gradient and surface effects on vibration and instability of nanotubes conveying nanoflow. Mechanics of Advanced Materials and Structures, 27, (7), (2020), pp. 586-598. https://doi.org/10.1080/15376494.2018.1487611.

[36] L. Li, Y. Hu, X. Li, and L. Ling. Size-dependent effects on critical flow velocity of fluidconveying microtubes via nonlocal strain gradient theory. Microfluidics and Nanofluidics, 20, (5), (2016), p. 76. https://doi.org/10.1007/s10404-016-1739-9.

[37] M. H. Ghayesh and A. Farajpour. Nonlinear mechanics of nanoscale tubes via nonlocal strain gradient theory. International Journal of Engineering Science, 129, (2018), pp. 84-95. https://doi.org/10.1016/j.ijengsci.2018.04.003.

[38] M. H. Ghayesh and A. Farajpour. Nonlinear coupled mechanics of nanotubes incorporating both nonlocal and strain gradient effects. Mechanics of Advanced Materials and Structures, 27, (5), (2020), pp. 373-382. https://doi.org/10.1080/15376494.2018.1473537.

[39] A. Azrar, M. Ben Said, L. Azrar, and A. A. Aljinaidi. Dynamic analysis of Carbon NanoTubes conveying fluid with uncertain parameters and random excitation. Mechanics of Advanced Materials and Structures, 26, (10), (2019), pp. 898-913. https://doi.org/10.1080/15376494.2018.1430272.

[40] L. Li, Y. Hu, and L. Ling. Wave propagation in viscoelastic single-walled carbon nanotubes with surface effect under magnetic field based on nonlocal strain gradient theory. Physica E: Low-dimensional Systems and Nanostructures, 75, (2016), pp. 118-124. https://doi.org/10.1016/j.physe.2015.09.028. 
[41] L. Li and Y. Hu. Wave propagation in fluid-conveying viscoelastic carbon nanotubes based on nonlocal strain gradient theory. Computational Materials Science, 112, (2016), pp. 282-288. https://doi.org/10.1016/j.commatsci.2015.10.044.

[42] Y. Zhen and L. Zhou. Wave propagation in fluid-conveying viscoelastic carbon nanotubes under longitudinal magnetic field with thermal and surface effect via nonlocal strain gradient theory. Modern Physics Letters B, 31, (08), (2017). https://doi.org/10.1142/s0217984917500695.

[43] N. D. Anh. Dual approach to averaged values of functions: A form for weighting coefficient. Vietnam Journal of Mechanics, 37, (2), (2015), pp. 145-150. https://doi.org/10.15625/08667136/37/2/6206.

[44] N. D. Anh, N. Q. Hai, and D. V. Hieu. The equivalent linearization method with a weighted averaging for analyzing of nonlinear vibrating systems. Latin American Journal of Solids and Structures, 14, (9), (2017), pp. 1723-1740. https://doi.org/10.1590/1679-78253488.

[45] D. V. Hieu. A new approximate solution for a generalized nonlinear oscillator. International Journal of Applied and Computational Mathematics, 5, (5), (2019). https://doi.org/10.1007/s40819-019-0709-9.

[46] D. V. Hieu and N. Q. Hai. Analyzing of nonlinear generalized duffing oscillators using the equivalent linearization method with a weighted averaging. Asian Research Journal of Mathematics, (2018), pp. 1-14. https://doi.org/10.9734/arjom/2018/40684.

[47] V. Hieu-Dang. An approximate solution for a nonlinear DuffingHarmonic oscillator. Asian Research Journal of Mathematics, (2019), pp. 1-14. https://doi.org/10.9734/arjom/2019/v15i430154.

[48] D. V. Hieu, N. Q. Hai, and D. T. Hung. The equivalent linearization method with a weighted averaging for solving undamped nonlinear oscillators. Journal of Applied Mathematics, 2018, (2018). https://doi.org/10.1155/2018/7487851.

[49] D. V. Hieu and N. Q. Hai. Free vibration analysis of quintic nonlinear beams using equivalent linearization method with a weighted averaging. Journal of Applied and Computational Mechanics, 5, (1), (2019), pp. 46-57. https://doi.org/10.22055/JACM.2018.24919.1217.

[50] S. S. Rao. Vibration of continuous systems. John Wiley \& Sons, Inc., (2007).

[51] M. Bayat, I. Pakar, and G. Domairry. Recent developments of some asymptotic methods and their applications for nonlinear vibration equations in engineering problems: A review. Latin American Journal of Solids and Structures, 9, (2), (2012), pp. 1-93. https://doi.org/10.1590/s1679-78252012000200003.

[52] T.-P. Chang. Nonlinear free vibration analysis of nanobeams under magnetic field based on nonlocal elasticity theory. Journal of Vibroengineering, 18, (3), (2016), pp. 1912-1919. https://doi.org/10.21595/jve.2015.16751. 